\title{
On the area discrepancy of triangulations of squares and trapezoids
}

\author{
Bernd Schulze* \\ Institute of Mathematics \\ Freie Universität Berlin \\ Arnimallee 2 \\ 14195 Berlin, Germany \\ bschulze@math.fu-berlin.de
}

Submitted: April 20, 2011; Accepted: Jun 16, 2011; Published: Jul 1, 2011

Mathematics Subject Classification: 52C15, 52B99

\begin{abstract}
In 1970 P. Monsky showed that a square cannot be triangulated into an odd number of triangles of equal areas; further, in 1990 E. A. Kasimatis and S. K. Stein proved that the trapezoid $T(\alpha)$ whose vertices have the coordinates $(0,0),(0,1)$, $(1,0)$, and $(\alpha, 1)$ cannot be triangulated into any number of triangles of equal areas if $\alpha>0$ is transcendental.

In this paper we first establish a new asymptotic upper bound for the minimal difference between the smallest and the largest area in triangulations of a square into an odd number of triangles. More precisely, using some techniques from the theory of continued fractions, we construct a sequence of triangulations $T_{n_{i}}$ of the unit square into $n_{i}$ triangles, $n_{i}$ odd, so that the difference between the smallest and the largest area in $T_{n_{i}}$ is $O\left(\frac{1}{n_{i}^{3}}\right)$.

We then prove that for an arbitrarily fast-growing function $f: \mathbb{N} \rightarrow \mathbb{N}$, there exists a transcendental number $\alpha>0$ and a sequence of triangulations $T_{n_{i}}$ of the trapezoid $T(\alpha)$ into $n_{i}$ triangles, so that the difference between the smallest and the largest area in $T_{n_{i}}$ is $O\left(\frac{1}{f\left(n_{i}\right)}\right)$.
\end{abstract}

Keywords: triangulation, equidissection, area discrepancy, square, trapezoid, continued fraction

\section{Introduction}

In this paper we consider simplicial triangulations of squares and trapezoids. By 'simplicial' we mean that the intersection of any two triangles in the triangulation, if non-empty,

*Supported by the DFG Research Unit 565 'Polyhedral Surfaces'. 
is either a common vertex or two vertices and the entire edge that joins them. In other words, a vertex is not allowed to lie in the interior of an edge of another triangle. However, we do allow vertices to lie on the edges of the square (or the trapezoid, respectively). Throughout the paper, by a triangulation we will always mean a simplicial triangulation.

It is a celebrated result of Paul Monsky that a square cannot be triangulated into an odd number of triangles of equal areas [11] (see also [1, 16]). Following Monsky's result, a number of authors have investigated the existence of 'equal-area triangulations' for various other types of polygons, such as trapezoids, regular $n$-gons, polyominos, etc. (see $[7,5,12,4,6,14]$, for example). See also [16] for a nice survey of some basic results in the theory. In recent years, research activities related to 'equal-area triangulations' of polygons have further increased due to some questions and conjectures posed by Richard Kenyon, Sherman Stein, and Günter M. Ziegler [10, 18, 15, 17, 2].

In Section 2 of this paper, we first address the following question asked by Günter M. Ziegler in 2003: given an odd number $n \in \mathbb{N}$, how small can the difference between the smallest and the largest area in a triangulation of a square into $n$ triangles become?

Formally, this problem may be described as follows. If for a triangulation $T_{n}$ of the unit square into $n$ triangles with areas $A_{1}, \ldots, A_{n}$, we define

$$
\operatorname{Max}\left(T_{n}\right):=\max _{1 \leq i<j \leq n}\left|A_{i}-A_{j}\right|,
$$

then we are interested in

$$
\mathrm{M}(n):=\min _{T_{n} \in S_{n}} \operatorname{Max}\left(T_{n}\right),
$$

where $S_{n}$ is the set of all triangulations of the unit square into $n$ triangles. It is easy to see that the minimum $\mathrm{M}(n)$ is in fact attained (see [10]). Obviously, we have $\mathrm{M}(n)=0$ if $n$ is even. So we suppose that $n$ is odd.

The following trivial - though currently best known - asymptotic upper bound for $\mathrm{M}(n)$ was established in [10]:

$$
\mathrm{M}(n)=O\left(\frac{1}{n^{2}}\right)
$$

In Section 2.2 (Theorem 2.5), we derive

$$
\mathrm{M}(n)=O\left(\frac{1}{n^{3}}\right)
$$

by constructing a sequence $\left\{T_{n_{i}}\right\}$ of triangulations of the unit square that satisfies $\operatorname{Max}\left(T_{n_{i}}\right)=O\left(\frac{1}{n_{i}^{3}}\right)$.

Some of the difficulties that arise in further improving this upper bound for $\mathrm{M}(n)$ are discussed in Section 2.3.

In Section 3, we study the area discrepancy of triangulations of trapezoids. For any real number $\alpha>0$, we let $T(\alpha)$ denote the trapezoid whose vertices have the coordinates $(0,0)$, $(0,1),(1,0)$, and $(\alpha, 1)$. Note that we may restrict our attention to such trapezoids, since any trapezoid is affinely equivalent to a trapezoid $T(\alpha)$. Analogously to the definitions above, we let

$$
\mathrm{M}(\alpha, n):=\min _{T_{n} \in S_{n}^{(\alpha)}} \operatorname{Max}\left(T_{n}\right)
$$


where $S_{n}^{(\alpha)}$ is the set of all triangulations of $T(\alpha)$ into $n$ triangles, and for any triangulation $T_{n}$ of $T(\alpha)$ into $n$ triangles with areas $A_{1}, \ldots, A_{n}, \operatorname{Max}\left(T_{n}\right)$ is defined as

$$
\operatorname{Max}\left(T_{n}\right):=\max _{1 \leq i<j \leq n}\left|A_{i}-A_{j}\right|
$$

It is well known that if $\alpha$ is transcendental, then $T(\alpha)$ cannot be triangulated into triangles of equal areas (see [7] as well as $[5,16,12,4]$, for example), so that for every $n \in \mathbb{N}$ we have

$$
\mathrm{M}(\alpha, n)>0 .
$$

One might suspect that - due to the large number of degrees of freedom for the vertex coordinates of a triangulation of a trapezoid (or, in particular, of a square) - there exists an exponential asymptotic upper bound for $\mathrm{M}(\alpha, n)$ (see also [10]). We prove in Section 3 (Theorem 3.2) that for suitable transcendental numbers $\alpha$, the following even stronger statement holds:

Given an (arbitrarily fast-growing) function $f: \mathbb{N} \rightarrow \mathbb{N}$, there exists a transcendental number $\alpha>0$ and a strictly monotone increasing sequence of natural numbers $n_{i}$ with

$$
M\left(\alpha, n_{i}\right)=O\left(\frac{1}{f\left(n_{i}\right)}\right)
$$

\section{Odd triangulations of a square}

\subsection{Preliminaries}

The starting point for our construction of sequences of triangulations which prove (1) are certain triangulations of a trapezoid, as they are described by Stein and Szabó in [16].

Theorem 2.1 [16] Let $t_{1}, t_{2}$, and $t_{3}$ be positive integers such that $t_{2}^{2}-4 t_{1} t_{3}$ is positive and is not the square of an integer (i.e., $f(x)=t_{3} x^{2}-t_{2} x+t_{1}$ has two positive nonrational roots). Let $c$ be a root of $f(x)$ and let $b=\frac{c t_{3}}{1+c t_{3}}$. Then

(i) $0<b<1$;

(ii) the triangulation of the trapezoid $A B C D$ into the triangles $\Delta_{1}, \Delta_{2}$, and $\Delta_{3}$ with respective areas $A_{1}, A_{2}$, and $A_{3}$ depicted in Figure 1 satisfies

$$
\frac{A_{2}}{A_{1}}=\frac{t_{2}}{t_{1}} \quad \text { and } \quad \frac{A_{3}}{A_{1}}=\frac{t_{3}}{t_{1}} .
$$

Corollary 2.2 [16] A triangulation of $\Delta_{1}$ into $t_{1}, \Delta_{2}$ into $t_{2}$, and $\Delta_{3}$ into $t_{3}$ triangles of equal areas gives rise to a triangulation of the trapezoid $A B C D$ into $t_{1}+t_{2}+t_{3}$ triangles of equal areas.

To prove (1) we need the following stronger version of Corollary 2.2: 


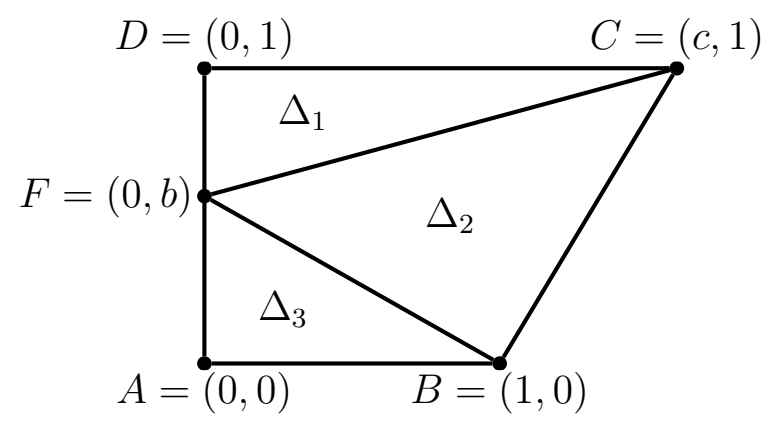

Figure 1: A triangulation of the trapezoid $A B C D$, where $b$ and $c$ are defined as in Theorem 2.1.

Corollary 2.3 Let $a \in \mathbb{N}$, and let $t_{1}, t_{2}$, and $t_{3}$ be as in Theorem 2.1. Then the following statements hold:

(i) If $t_{2}$ is odd, then the trapezoid $A B C D$ in Figure 1 can be triangulated into a $\left(t_{1}+t_{2}+\right.$ $\left.t_{3}\right)$ triangles of equal areas, so that no vertex lies in the interior of the line segment $\overline{B C}$;

(ii) if $t_{2}$ is even, then the trapezoid $A B C D$ in Figure 1 can be triangulated into a $\left(t_{1}+\right.$ $\left.t_{2}+t_{3}\right)$ triangles of equal areas, so that one of the vertices of the triangles is the midpoint of the line segment $\overline{B C}$ and no other vertices lie in the interior of $\overline{B C}$.

Proof. ( $i$ ) Let $a=2^{\alpha} a^{\prime}$, where $a^{\prime}$ is odd and $\alpha \geq 0$. It is easy to triangulate $\Delta_{3}$ into $a t_{3}$ triangles of equal areas by placing $a t_{3}-1$ vertices equidistantly on the line segment $\overline{A B}$. Then we triangulate each of the triangles $\Delta_{1}$ and $\Delta_{2}$ into $2^{\alpha}$ triangles by placing $2^{\alpha}-1$ vertices equidistantly on the line segment $\overline{F C}$. Since $t_{2} a^{\prime}$ is odd, we can triangulate each of the triangles in the resulting triangulation of $\Delta_{2}$ into $t_{2} a^{\prime}$ triangles of equal areas without placing vertices on edges. If $t_{1}$ is odd, the same can be done with the triangulation of $\Delta_{1}$, yielding a desired triangulation of the trapezoid $A B C D$. If $t_{1}$ is even, then we denote the vertices that were added on the line segment $\overline{F C}$ by $V_{1}, \ldots, V_{2^{\alpha}-1}$, and triangulate each of the $2^{\alpha}$ triangles in the triangulation of $\Delta_{1}$ into $t_{1} a^{\prime}$ triangles of equal areas by placing $t_{1} a^{\prime}-1$ vertices equidistantly on each of the line segments $\overline{D V_{2 i-1}}, i=1, \ldots, 2^{\alpha-1}$. This proves $(i)$.

(ii) Let $t_{2}=2^{\tau} t^{\prime}$, where $t^{\prime}$ is odd and $\tau \geq 1$. Then we triangulate the triangle $\Delta_{2}$ as follows. First, we split $\Delta_{2}$ into two triangles of equal areas by connecting the vertex $F$ with the midpoint $M$ of the line segment $\overline{B C}$. Then we triangulate each of these two triangles into $2^{\tau-1} t^{\prime} a$ triangles of equal areas by placing $2^{\tau-1} t^{\prime} a-1$ vertices equidistantly on the line segment $\overline{F M}$. The triangles $\Delta_{1}$ and $\Delta_{3}$ we triangulate into $a t_{1}$ and $a t_{3}$ triangles of equal areas by placing $a t_{1}-1$ and $a t_{3}-1$ vertices equidistantly on the line segments $\overline{A B}$ and $\overline{D C}$, respectively. This yields a desired triangulation of the trapezoid $A B C D$.

Throughout this paper, we will need good rational approximations of a real number $\alpha$; so we will frequently use some basic results from the theory of continued fractions which 
we summarize in Theorem 2.4. Good sources for these results are [8, 9], for example.

Let the continued fraction representation of a real number $\alpha>0$ be given by

$$
\alpha=\left[a_{1}, a_{2}, a_{3}, \ldots\right]:=a_{1}+\frac{1}{a_{2}+\frac{1}{a_{3}+\frac{1}{a_{4}+\ldots}}},
$$

where $a_{1} \in \mathbb{N} \cup\{0\}$ and $a_{i} \in \mathbb{N}$ for all $i \geq 1$. Then the rational number

$$
\left[a_{1}, a_{2}, \ldots, a_{n}\right]:=a_{1}+\frac{1}{a_{2}+\ldots+\frac{1}{a_{n-1}+\frac{1}{a_{n}}}}
$$

is called the $n$th convergent of $\alpha$.

Theorem 2.4 Let $\alpha \in \mathbb{R}, \alpha>0$, and let $\frac{p_{n}}{q_{n}}$ be the nth convergent of $\alpha$ with $\operatorname{gcd}\left(p_{n}, q_{n}\right)=$ 1. Then

(i) the process of representing $\alpha$ as a continued fraction terminates if and only if $\alpha$ is rational;

(ii) $p_{n} q_{n-1}-p_{n-1} q_{n}=(-1)^{n}$;

(iii) $\left|\alpha-\frac{p_{n}}{q_{n}}\right| \leq \frac{1}{q_{n}^{2}}$

\subsection{The main result for the square}

Theorem 2.5 Let $T_{n_{0}}^{(1)}, T_{n_{0}}^{(2)}$, and $T_{n_{0}}^{(3)}$ be the triangulations of the rectangle AECD depicted in Figure 2 with $E=(c, 0), G=\left(1+\frac{2}{3}(c-1), 0\right)$, and $M=\left(1+\frac{1}{2}(c-1), \frac{1}{2}\right)$; these triangulations extend the triangulation of the trapezoid $A B C D$ in Figure 1. Then for some $k \in\{1,2,3\}$, there exists a sequence of triangulations $T_{n_{i}}^{(k)}, i \geq 0$, of $A E C D$ into $n_{i}$ triangles so that

(i) $n_{0}<n_{1}<n_{2}<\ldots \quad\left(n_{i}\right.$ odd for $\left.i \geq 1\right)$;

(ii) $T_{n_{i}}^{(k)}$ is a refinement of the triangulation $T_{n_{0}}^{(k)}$ (i.e., each triangle of $T_{n_{i}}^{(k)}$ is fully contained in a triangle of $\left.T_{n_{0}}^{(k)}\right)$;

(iii) $\operatorname{Max}\left(T_{n_{i}}^{(k)}\right)=O\left(\frac{1}{n_{i}^{3}}\right)$.

Remark 2.1 By appropriately scaling the x-axis, Theorem 2.5 can immediately be transferred from the rectangle AECD to the unit square.

Proof of Theorem 2.5. Wlog we assume that $c>1$ (as it is the case in Figures 1 $2)$. For $c<1$, the proof proceeds analogously. Let $A_{\text {trap }}$ denote the area of the trapezoid $A B C D$ and $A_{\text {tria }}$ denote the area of the triangle $B E C$. Then we have

$$
\frac{A_{\text {trap }}}{A_{\text {tria }}}=\frac{c+1}{c-1}
$$

THE ELECTRONiC JOURNAL OF COMBINATORICs 18 (2011), \#P137 


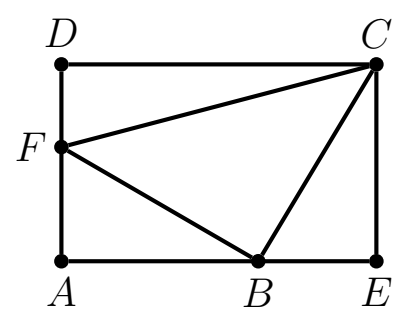

(a)

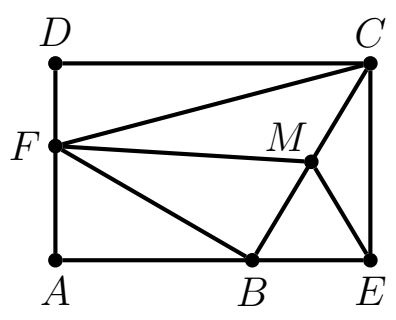

(b)

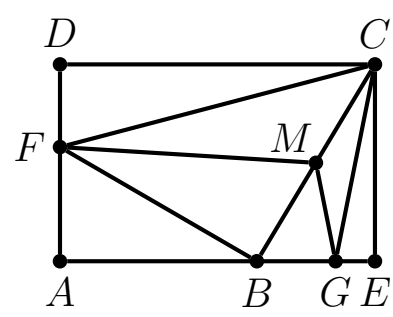

(c)

Figure 2: Triangulations of the rectangle $A E C D$ : (a) the triangulation $T_{n_{0}}^{(1)}$; (b) the triangulation $T_{n_{0}}^{(2)}$; (c) the triangulation $T_{n_{0}}^{(3)}$.

and since $c \notin \mathbb{Q}, \frac{A_{\text {trap }}}{A_{\text {tria }}}$ is not rational. We now consider four cases.

Case 1 (see Figure $2(a)$ ): Suppose that both $t_{2}$ and $t_{1}+t_{2}+t_{3}$ are odd. By Theorem $2.4(i i i)$, for the $n$th convergent $\frac{p_{n}}{q_{n}}$ of $\frac{A_{\text {trap }}}{A_{\text {tria }}}$, we have

$$
\left|\frac{A_{\text {trap }}}{A_{\text {tria }}}-\frac{p_{n}}{q_{n}}\right| \leq \frac{1}{q_{n}^{2}}
$$

and hence

$$
\left|\frac{A_{\text {trap }}}{p_{n}}-\frac{A_{\text {tria }}}{q_{n}}\right| \leq \frac{A_{\text {tria }}}{p_{n}} \cdot \frac{1}{q_{n}^{2}} .
$$

By Theorem 2.4 (iii), there exist positive constants $c_{1}$ and $c_{2}$ such that for all $n \in \mathbb{N}$ we have

$$
c_{1} q_{n} \leq p_{n} \leq c_{2} q_{n}
$$

Therefore,

$$
\left|\frac{A_{\text {trap }}}{\left(t_{1}+t_{2}+t_{3}\right) p_{n}}-\frac{A_{\text {tria }}}{\left(t_{1}+t_{2}+t_{3}\right) q_{n}}\right| \leq \frac{A_{\text {tria }}}{c_{1}^{\prime}} \cdot \frac{1}{q_{n}^{3}}
$$

where $c_{1}^{\prime}=\left(t_{1}+t_{2}+t_{3}\right) c_{1}$.

By Corollary $2.3(i)$, the trapezoid $A B C D$ can be triangulated into $\left(t_{1}+t_{2}+t_{3}\right) p_{n}$ triangles of equal areas, and the triangle $B E C$ can be triangulated into $\left(t_{1}+t_{2}+t_{3}\right) q_{n}$ triangles of equal areas, so that we obtain a triangulation $T_{n_{i}}^{(1)}$ of the rectangle $A E C D$ into $n_{i}=\left(t_{1}+t_{2}+t_{3}\right)\left(p_{n}+q_{n}\right)$ triangles with

$$
\operatorname{Max}\left(T_{n_{i}}^{(1)}\right) \leq \frac{A_{\text {tria }}}{c_{1}^{\prime}} \cdot \frac{1}{q_{n}^{3}} .
$$

It follows from (3) that the number $n_{i}$ of triangles in $T_{n_{i}}^{(1)}$ is at most $\left(t_{1}+t_{2}+t_{3}\right)\left(c_{2}+1\right) q_{n}$. Moreover, $n_{i}$ is odd for infinitely many $n_{i}$, because if $p_{n}+q_{n}$ is even, then it follows from $\operatorname{gcd}\left(p_{n}, q_{n}\right)=1$ that both $p_{n}$ and $q_{n}$ are odd, so that, by Theorem $2.4(i i), p_{n-1}+q_{n-1}$ is odd. Thus, there exists a sequence $\left\{T_{n_{i}}^{(1)}\right\}_{i \geq 0}$ of triangulations of $A E C D$ which satisfies the desired properties. 
Case 2 (see again Figure $2(a)$ ): Suppose that $t_{2}$ is odd and that $t_{1}+t_{2}+t_{3}$ is even. By Theorem $2.4\left(\right.$ iii), for the $n$th convergent $\frac{p_{n}}{q_{n}}$ of

$$
\frac{\frac{A_{\text {trap }}}{t_{1}+t_{2}+t_{3}}}{A_{\text {tria }}}
$$

we have

$$
\left|\frac{A_{\text {trap }}}{\left(t_{1}+t_{2}+t_{3}\right) p_{n}}-\frac{A_{\text {tria }}}{q_{n}}\right| \leq \frac{A_{\text {tria }}}{p_{n}} \cdot \frac{1}{q_{n}^{2}} .
$$

Thus, analogously to Case 1, Corollary $2.3(i)$ guarantees the existence of a triangulation $T_{n_{i}}^{(1)}$ of the rectangle $A E C D$ into $n_{i}=\left(t_{1}+t_{2}+t_{3}\right) p_{n}+q_{n}$ triangles with

$$
\operatorname{Max}\left(T_{n_{i}}^{(1)}\right) \leq c \cdot \frac{1}{q_{n}^{3}}
$$

for some constant $c$. Since, by Theorem $2.4(i i), q_{n}$ and $q_{n-1}$ cannot both be even, $n_{i}$ is odd for infinitely many $n_{i}$. Thus, there exists a sequence $\left\{T_{n_{i}}^{(1)}\right\}_{i \geq 0}$ of triangulations of $A E C D$ which satisfies the desired properties.

Case 3 (see Figure 2 (b)): Suppose that $t_{2}$ is even and that $t_{1}+t_{2}+t_{3}$ is odd. Note that in the triangulation $T_{n_{0}}^{(2)}$ of $A E C D$ depicted in Figure $2(\mathrm{~b})$, the triangle $B E C$ is triangulated into two triangles of equal areas. By Theorem $2.4($ iii $)$, for the $n$th convergent $\frac{p_{n}}{q_{n}}$ of

$$
\frac{A_{\text {trap }}}{\frac{t_{1}+t_{2}+t_{3}}{A_{t r i a}}}
$$

we have

$$
\left|\frac{A_{\text {trap }}}{\left(t_{1}+t_{2}+t_{3}\right) p_{n}}-\frac{A_{\text {tria }}}{2 q_{n}}\right| \leq \frac{A_{\text {tria }}}{2 p_{n}} \cdot \frac{1}{q_{n}^{2}} .
$$

Thus, it follows from Corollary $2.3(i i)$ that there exists a triangulation $T_{n_{i}}^{(2)}$ of the rectangle $A E C D$ into $n_{i}=\left(t_{1}+t_{2}+t_{3}\right) p_{n}+2 q_{n}$ triangles with

$$
\operatorname{Max}\left(T_{n_{i}}^{(2)}\right) \leq c \cdot \frac{1}{q_{n}^{3}}
$$

for some constant $c$. Since, by Theorem $2.4(i i), p_{n}$ and $p_{n-1}$ cannot both be even, $n_{i}$ is odd for infinitely many $n_{i}$. Thus, there exists a sequence $\left\{T_{n_{i}}^{(2)}\right\}_{i \geq 0}$ of triangulations of $A E C D$ which satisfies the desired properties.

Case 4 (see Figure 2 (c)): Finally, suppose that both $t_{2}$ and $t_{1}+t_{2}+t_{3}$ are even. Note that in the triangulation $T_{n_{0}}^{(3)}$ of $A E C D$ depicted in Figure 2 (c), the triangle $B E C$ is triangulated into three triangles of equal areas. By Theorem $2.4(i i i)$, for the $n$th convergent $\frac{p_{n}}{q_{n}}$ of

$$
\frac{\frac{A_{\text {trap }}}{t_{1}+t_{2}+t_{3}}}{\frac{A_{t r i a}}{3}}
$$


we have

$$
\left|\frac{A_{\text {trap }}}{\left(t_{1}+t_{2}+t_{3}\right) p_{n}}-\frac{A_{\text {tria }}}{3 q_{n}}\right| \leq \frac{A_{\text {tria }}}{3 p_{n}} \cdot \frac{1}{q_{n}^{2}} .
$$

Thus, by Corollary $2.3(\mathrm{ii})$, there exists a triangulation $T_{n_{i}}^{(3)}$ of the rectangle $A E C D$ into $n_{i}=\left(t_{1}+t_{2}+t_{3}\right) p_{n}+3 q_{n}$ triangles with

$$
\operatorname{Max}\left(T_{n_{i}}^{(3)}\right) \leq c \cdot \frac{1}{q_{n}^{3}}
$$

for some constant $c$. Further, we again have that $n_{i}$ is odd for infinitely many $n_{i}$, since, by Theorem $2.4(i i), q_{n}$ and $q_{n-1}$ cannot both be even. Thus, there exists a sequence $\left\{T_{n_{i}}^{(3)}\right\}_{i \geq 0}$ of triangulations of $A E C D$ which satisfies the desired properties. This completes the proof.

\section{$2.3 \quad$ Further remarks}

In the previous section (Theorem 2.5) we showed that $\mathrm{M}(n)=O\left(\frac{1}{n^{3}}\right)$ by constructing a sequence $\left\{T_{n_{i}}\right\}$ of triangulations of the unit square, starting from a suitable triangulation $T_{n_{0}}$, with the property that each triangulation $T_{n_{i}}$ is a refinement of the triangulation $T_{n_{0}}$. Can the asymptotic upper bound $O\left(\frac{1}{n^{3}}\right)$ for $\mathrm{M}(n)$ be further improved with this method?

Clearly, if the triangles $\Delta_{1}, \ldots, \Delta_{n_{0}}$ of $T_{n_{0}}$ with respective areas $A_{1}, \ldots, A_{n_{0}}$ satisfy the property that all quotients $\frac{A_{i}}{A_{1}}, i=2, \ldots, n_{0}$, are rational, then one cannot obtain an analogous result to Theorem 2.5 by refining $T_{n_{0}}$, because rational numbers have finite continued fraction representations (recall Theorem $2.4(i)$ ) and $\left|\alpha-\frac{p}{q}\right|<\frac{1}{q^{2}}$ has only a finite number of solutions if $\alpha$ is rational (see [3], for example).

Our analyses in the previous sections suggest to consider triangulations of the following type:

Definition 2.1 We say that a triangulation $T_{n_{0}}$ of the unit square (or, more generally, of a trapezoid) into triangles $\Delta_{1}, \ldots, \Delta_{n_{0}}$ is an $r$-triangulation if for any natural numbers $B_{1}, \ldots, B_{n_{0}}$, there exists a natural number $B$ and a refinement of $T_{n_{0}}$ in which each $\Delta_{i}$ is triangulated into $B \cdot B_{i}$ triangles of equal areas. (See also Remark 3.1 for further comments on r-triangulations.)

Remark 2.2 Let $T_{n_{0}}$ be a triangulation of the unit square whose triangles $\Delta_{1}, \ldots, \Delta_{n_{0}}$ have respective areas $A_{1}, \ldots, A_{n_{0}}$. If $T_{n_{0}}$ is an r-triangulation and all quotients $\frac{A_{i}}{A_{1}}, i=$ $2, \ldots, n_{0}$, are rational, then $T_{n_{0}}$ can of course be refined to a triangulation of the unit square whose triangles all have equal areas. However, it then follows from Monsky's theorem (see [11]) that the number of triangles in this triangulation must be even.

Remark 2.3 To improve the asymptotic upper bound for $M(n)$ in Theorem 2.5 it is natural to try the following approach.

Let $A_{1}, \ldots, A_{n_{0}}$ be the areas of the triangles $\Delta_{1}, \ldots, \Delta_{n_{0}}$ of an r-triangulation $T_{n_{0}}$ of the unit square, and let $A_{1}^{\prime}, \ldots, A_{n_{0}}^{\prime}$ be the areas of the triangles $\Delta_{1}^{\prime}, \ldots, \Delta_{n_{0}}^{\prime}$ of a 
triangulation $T_{n_{0}}^{\prime}$ of the unit square, where the coordinates of the vertices of the $\Delta_{i}^{\prime}$ are rational numbers that approximate the coordinates of the vertices of the $\Delta_{i}$ very well. Moreover, the combinatorial type of the triangulations $T_{n_{0}}$ and $T_{n_{0}}^{\prime}$ shall be the same. Then the quotients $\frac{A_{i}^{\prime}}{A_{1}^{\prime}}, i=2, \ldots, n_{0}$, are of course rational, say

$$
\frac{A_{i}^{\prime}}{A_{1}^{\prime}}=\frac{a_{i}}{a_{1}} \quad \text { with } a_{i} \in \mathbb{N} \text { for all } i
$$

Due to the continuity of the area function, the approximation

$$
\left|\frac{A_{i}}{A_{1}}-\frac{a_{i}}{a_{1}}\right|
$$

is then also very good. It is therefore natural to refine the triangulation $T_{n_{0}}$ by triangulating each $\Delta_{i}$ into $B a_{i}$ triangles of equal areas. This yields a triangulation with $B\left(a_{1}+\ldots+a_{n_{0}}\right)$ triangles.

Unfortunately, $B\left(a_{1}+\ldots+a_{n_{0}}\right)$ will always be even, because it follows from (4) that if each triangle $\Delta_{i}^{\prime}$ is triangulated into $B a_{i}$ triangles of equal areas, then one obtains a triangulation of the unit square whose triangles have all equal areas.

The next theorem (Theorem 2.7) shows that if there exist two triangles in $T_{n_{0}}$ whose ratio of areas is not rational but algebraic over $\mathbb{Q}$, then Theorem 2.5 can also not be improved by refining $T_{n_{0}}$. This result is based on the following well-known fact:

Lemma 2.6 (Thue, Siegel, Roth) [13] Let $\epsilon>0, A>0$, and $\alpha \in \mathbb{R}$ be nonrational, but algebraic over $\mathbb{Q}$. Then there only exist finitely many fractions $\frac{p}{q}, \operatorname{gcd}(p, q)=1$, with

$$
\left|\alpha-\frac{p}{q}\right|<\frac{A}{q^{2+\epsilon}}
$$

Theorem 2.7 Let $T_{n_{0}}$ be a triangulation of the unit square which contains two triangles $\Delta_{1}$ and $\Delta_{2}$ with respective areas $A_{1}$ and $A_{2}$ so that

$$
\alpha=\frac{A_{1}}{A_{2}}
$$

is not rational, but algebraic over $\mathbb{Q}$. Let $\epsilon>0$. Then there exists no sequence of triangulations $T_{n_{i}}, i \geq 0$, of the unit square into $n_{i}$ triangles with

(i) $n_{0}<n_{1}<n_{2}<\ldots$;

(ii) $T_{n_{i}}$ is a refinement of the triangulation $T_{n_{0}}$ (i.e., each triangle of $T_{n_{i}}$ is fully contained in a triangle of $\left.T_{n_{0}}\right)$;

(iii) $\operatorname{Max}\left(T_{n_{i}}\right)=O\left(\frac{1}{n_{i}^{3+\epsilon}}\right)$. 
Proof. Let $\left\{T_{n_{i}}\right\}_{i \geq 0}$ be a sequence of triangulations satisfying the conditions $(i)$, (ii), and (iii). Then $\left\{T_{n_{i}}\right\}_{i \geq 0}$ gives rise to sequences $\left\{T_{n_{i}^{\prime}}\right\}_{i \geq 0}$ and $\left\{T_{n_{i}^{\prime \prime}}\right\}_{i \geq 0}$ of triangulations of the triangles $\Delta_{1}$ and $\Delta_{2}$ into $n_{i}^{\prime}$ and $n_{i}^{\prime \prime}$ triangles, respectively. Condition (iii) implies that $\lim _{i \rightarrow \infty} n_{i}^{\prime}=\infty$ and $\lim _{i \rightarrow \infty} n_{i}^{\prime \prime}=\infty$. So, wlog, the sequence $\left\{T_{n_{i}}\right\}_{i \geq 0}$ can be chosen so that

$$
\begin{aligned}
& n_{0}^{\prime} \leq n_{1}^{\prime} \leq n_{2}^{\prime} \leq \ldots \\
& n_{0}^{\prime \prime} \leq n_{1}^{\prime \prime} \leq n_{2}^{\prime \prime} \leq \ldots
\end{aligned}
$$

Note that there exist triangles $D_{1}$ and $D_{2}$ in the triangulations $T_{n_{i}^{\prime}}$ and $T_{n_{i}^{\prime \prime}}$, respectively, so that the difference between the area of $D_{1}$ and the area of $D_{2}$ is at least

$$
\left|\frac{A_{1}}{n_{i}^{\prime}}-\frac{A_{2}}{n_{i}^{\prime \prime}}\right|
$$

because the maximum over all differences between the area of a triangle in $T_{n_{i}^{\prime}}$ and the area of a triangle in $T_{n_{i}^{\prime \prime}}$ is minimal if both $\Delta_{1}$ and $\Delta_{2}$ are triangulated into triangles of equal areas. Thus, we have

$$
\operatorname{Max}\left(T_{n_{i}}\right) \geq\left|\frac{A_{1}}{n_{i}^{\prime}}-\frac{A_{2}}{n_{i}^{\prime \prime}}\right| .
$$

By the definition of $\alpha$, we have

$$
\left|\frac{A_{1}}{n_{i}^{\prime}}-\frac{A_{2}}{n_{i}^{\prime \prime}}\right|=\left|\alpha-\frac{n_{i}^{\prime}}{n_{i}^{\prime \prime}}\right| \cdot \frac{A_{2}}{n_{i}^{\prime}} .
$$

Now, if condition (iii) holds, then it follows from (5) that there exists a constant $c>0$ with

$$
\left|\frac{A_{1}}{n_{i}^{\prime}}-\frac{A_{2}}{n_{i}^{\prime \prime}}\right| \leq \frac{c}{n_{i}^{3+\epsilon}} \quad \text { for all } i \in \mathbb{N} .
$$

Therefore, by (6) and (7), we have

$$
\left|\alpha-\frac{n_{i}^{\prime}}{n_{i}^{\prime \prime}}\right| \leq \frac{n_{i}^{\prime}}{A_{2}} \cdot \frac{c}{n_{i}^{3+\epsilon}} \leq \frac{c}{A_{2}} \cdot \frac{1}{n_{i}^{2+\epsilon}} \leq \frac{c}{A_{2}} \cdot \frac{1}{n_{i}^{\prime \prime 2+\epsilon}} \quad \text { for all } i \in \mathbb{N} .
$$

If $\frac{n_{i}^{\prime}}{n_{i}^{\prime \prime}}$ takes on infinitely many different values, then (8) contradicts Lemma 2.6.

So, suppose there exists an $i \in \mathbb{N}$, so that

$$
\frac{n_{j}^{\prime}}{n_{j}^{\prime \prime}}=\frac{k_{j} n_{i}^{\prime}}{k_{j} n_{i}^{\prime \prime}}
$$

for infinitely many $j$ with $j \geq i$, where $k_{j} \in \mathbb{N}, k_{j} \geq 0$. Then it follows from (5) that for infinitely many $j$ with $j \geq i$, we have

$$
\operatorname{Max}\left(T_{n_{j}}\right) \geq \frac{1}{k_{j}} \cdot\left|\frac{A_{1}}{n_{i}^{\prime}}-\frac{A_{2}}{n_{i}^{\prime \prime}}\right| .
$$


Further, we have $n_{j} \geq k_{j}\left(n_{i}^{\prime}+n_{i}^{\prime \prime}\right)$, and hence

$$
\operatorname{Max}\left(T_{n_{j}}\right) \cdot n_{j} \geq\left|\frac{A_{1}}{n_{i}^{\prime}}-\frac{A_{2}}{n_{i}^{\prime \prime}}\right| \cdot\left(n_{i}^{\prime}+n_{i}^{\prime \prime}\right)
$$

for infinitely many $j$ with $j \geq i$. This contradicts $(i i i)$, since the right-hand side of the above inequality is constant.

Theorem 2.8 The condition in Theorem 2.7 that the triangulation $T_{n_{0}}$ contains two triangles $\Delta_{1}$ and $\Delta_{2}$ with respective areas $A_{1}$ and $A_{2}$ so that $\alpha=\frac{A_{1}}{A_{2}}$ is not rational but algebraic over $\mathbb{Q}$ may be replaced by the condition that $T_{n_{0}}$ contains a triangle $\Delta_{1}$ whose area $A_{1}$ is not rational but algebraic over $\mathbb{Q}$.

Proof. If we assume that there exists a sequence of triangulations of the unit square starting with $T_{n_{0}}$ and satisfying the conditions $(i)-(i i i)$ of Theorem 2.7 , then, analogously to the proof of Theorem 2.7, it follows that for each of the triangles $\Delta_{j}, j=2, \ldots, n_{0}$, of the triangulation $T_{n_{0}}$, there exists a sequence $\left(n_{j_{i}}\right)_{i \in \mathbb{N}}$ of natural numbers, so that for appropriate positive constants $c_{2}, \ldots, c_{n_{0}}$, we have

$$
\left|\frac{A_{j}}{A_{1}}-\frac{n_{j_{i}}}{n_{1_{i}}}\right| \leq c_{j} \cdot \frac{1}{n_{1_{i}}^{2+\epsilon}} \quad \text { for all } i \in \mathbb{N},
$$

where $A_{j}$ is the area of the triangle $\Delta_{j}$ for each $j$. Further, it follows from the proof of Theorem 2.7 that for each $j=1, \ldots, n_{0}, \frac{n_{j_{i}}}{n_{1_{i}}}$ takes on infinitely many different values. It follows that the same is also true for $n_{1_{i}}$, for otherwise (9) would not hold for all $i \in \mathbb{N}$.

Since we have $A_{2}+\ldots+A_{n_{0}}=1-A_{1}$, summation of the inequalities in (9) yields a constant $c>0$ and a sequence $\left(n_{i}\right)_{i \in \mathbb{N}}$ with

$$
\left|\frac{1-A_{1}}{A_{1}}-\frac{n_{i}}{n_{1_{i}}}\right| \leq c \cdot \frac{1}{n_{1_{i}}^{2+\epsilon}} \quad \text { for all } i \in \mathbb{N} .
$$

Since, by assumption, $A_{1}$ is not rational but algebraic over $\mathbb{Q}$, the same is also true for $\frac{1-A_{1}}{A_{1}}$. Moreover, since $n_{1_{i}}$ takes on infinitely many different values, the right-hand side of the inequality (10) becomes arbitrarily small as $i$ goes to infinity, and hence $\frac{n_{i}}{n_{1_{i}}}$ also takes on infinitely many different values. Thus, the inequality (10) contradicts Lemma 2.6.

It follows from Theorem 2.7 and the comments in the beginning of this section that an improvement of Theorem 2.5 using refinements of a given triangulation $T_{n_{0}}$ is only possible if the quotients $\frac{A_{i}}{A_{1}}, i=2, \ldots, n_{0}$, are all either rational or transcendental, and $\frac{A_{i}}{A_{1}}$ is transcendental for at least one $i$.

Note that a triangulation of the unit square into $n$ triangles is determined by the $(n-2)$ 'free' coordinates of the corresponding vertices. If $(n-2)$ of the $(n-1)$ quotients $\frac{A_{i}}{A_{1}}$ are rational, then the free coordinates satisfy $(n-2)$ polynomial equations with integer coefficients, so that one may suspect that all the free coordinates - and hence also all the quotients $\frac{A_{i}}{A_{1}}$ - are algebraic over $\mathbb{Q}$. In other words, we anticipate that an improvement of Theorem 2.5 can only be obtained if at least two of the quotients $\frac{A_{i}}{A_{1}}$ are transcendental. 
So, let $\frac{A_{2}}{A_{1}}, \ldots, \frac{A_{r+1}}{A_{1}}, r \geq 2$, be transcendental, and let $\frac{A_{r+2}}{A_{1}}, \ldots, \frac{A_{n}}{A_{1}}$ be rational. To improve Theorem 2.7 we then need simultaneous approximations of the form

$$
\left|\frac{A_{i}}{A_{1}}-\frac{a_{i}}{a_{1}}\right| \leq \frac{c}{a_{1}^{2+\epsilon}}, \quad i=2, \ldots, r+1,
$$

where $\epsilon>0$ and $c$ is a constant, so that $a_{1}+\ldots+a_{n}$ is odd. It remains open whether there exists such an example.

It is a well known fact (see [3], for example) that there always exists an approximation of the form

$$
\left|\frac{A_{i}}{A_{1}}-\frac{a_{i}}{a_{1}}\right| \leq \frac{c}{a_{1}^{1+\frac{1}{r}}}, \quad i=2, \ldots, r+1,
$$

where $c$ is a constant, but such an approximation is of course not good enough.

If, on the other hand, the approximation in (11) is 'too good', then $a_{1}+\ldots+a_{n}$ is surely even, since, by Monsky's theorem, the area discrepancy is strictly positive for all odd triangulations of the unit square.

\section{Triangulations of trapezoids}

While it seems to be difficult to further improve the asymptotic upper bound for $M(n)$ given in Theorem 2.5 using the refinement methods of the previous section, we can use the basic idea of Remark 2.3 to show a surprisingly strong result concerning the area discrepancy of triangulations of certain trapezoids (see Theorem 3.2).

Recall from Section 1 that for any real number $\alpha>0, T(\alpha)$ is the trapezoid whose vertices have the coordinates $(0,0),(0,1),(1,0)$, and $(\alpha, 1)$, and that, as shown in [7], we have

$$
\mathrm{M}(\alpha, n)>0
$$

for every $n \in \mathbb{N}$, whenever $\alpha>0$ is transcendental. To prove (2), we need the following well-known result (see [9]):

Lemma 3.1 Let $a_{1}, a_{2}, \ldots$ be natural numbers and $\alpha=\left[a_{1}, a_{2}, \ldots\right]$; further, let $\frac{p_{n}}{q_{n}}=$ $\left[a_{1}, \ldots, a_{n}\right]$ be the $n$th convergent of $\alpha$, where $\operatorname{gcd}\left(p_{n}, q_{n}\right)=1$. Then we have

(i) $\left|\alpha-\frac{p_{n}}{q_{n}}\right| \leq \frac{1}{q_{n}\left(a_{n+1} q_{n}+q_{n-1}\right)} \quad(n>1)$;

(ii) $\alpha$ is transcendental if there exists an $n_{0} \in \mathbb{N}$, so that for all $n \geq n_{0}$, we have $a_{n+1}>q_{n}^{n-1}$.

We also need the following remark concerning r-triangulations (recall Definition 2.1):

Remark 3.1 Let $T(\alpha), \alpha>0$, be a trapezoid. If $T_{n_{0}}$ is a triangulation of $T(\alpha)$ with the property that edges of triangles can be removed in such a way that one obtains a dissection of $T(\alpha)$ into quadrilaterals and triangles, where each triangle has at least one edge that lies on an edge of $T(\alpha)$, then $T_{n_{0}}$ is an r-triangulation. 
Proof. For triangles that have at least one of their edges, say $e$, on an edge of $T(\alpha)$, the desired dissection can trivially be obtained by adding points appropriately on $e$. By assumption, we can identify pairs of edge-sharing triangles in $T_{n_{0}}$, so that each of the remaining triangles has the property that at least one of its edges lies on an edge of $T(\alpha)$. Let $\Delta_{i}=A B C$ and $\Delta_{j}=B D C$ be such a pair of edge-sharing triangles (see Figure 3 ). We add the midpoint $P$ of the shared edge $B C$ as a new vertex. The edges $A P$ and $D P$

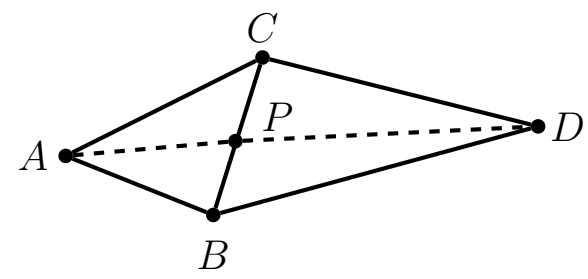

Figure 3: The edge-sharing triangles $A B C$ and $B D C$ in $T_{n_{0}}$.

triangulate each of the triangles $A B C$ and $B D C$ into two triangles of equal areas. By appropriately choosing $B_{i}-1$ points on $A P$, the triangle $\Delta_{i}$ can be triangulated into $2 B_{i}$ triangles of equal areas. Analogously, one obtains a triangulation of $\Delta_{j}$ into $2 B_{j}$ triangles of equal areas. This yields the desired refinement of $T_{n_{0}}$ for $B=2$.

It remains open, whether there exist triangulations which are not r-triangulations, or whether there exist triangulations which do not satisfy the conditions in Remark 3.1.

We are now ready to prove (2).

Theorem 3.2 Let $f: \mathbb{N} \rightarrow \mathbb{N}$ be an (arbitrarily fast-growing) function. Then there exists a transcendental number $\alpha>0$ and a strictly monotone increasing sequence of natural numbers $n_{i}$ with

$$
M\left(\alpha, n_{i}\right)=O\left(\frac{1}{f\left(n_{i}\right)}\right)
$$

Proof. For $\beta \in \mathbb{R}$, we denote $p(\beta)$ to be the point in $\mathbb{R}^{2}$ with the coordinates $(\beta, 1)$. Let $\alpha^{\prime}$ be a positive real number and $T_{n_{0}}^{\prime}$ be an r-triangulation of the trapezoid $T\left(\alpha^{\prime}\right)$ into $n_{0}$ triangles, so that none of the vertices of the triangles in $T_{n_{0}}^{\prime}$ lies on the edge between the points $p\left(\alpha^{\prime}\right)$ and $(1,0)$. By Remark 3.1, such a triangulation of $T\left(\alpha^{\prime}\right)$ clearly exists. In the following, we denote $p_{2}^{\prime}=(1,0)$ and we let $p\left(\alpha^{\prime}\right), p_{2}^{\prime}, \ldots, p_{m}^{\prime}$ be the points in $\mathbb{R}^{2}$ that correspond to the vertices of the triangles in $T_{n_{0}}^{\prime}$.

We show that there exists a transcendental number $\alpha>0$ and a triangulation $T_{n_{0}}$ of $T(\alpha)$, so that

(i) $T_{n_{0}}$ has the same combinatorial type as $T_{n_{0}}^{\prime}$;

(ii) there exists a sequence of triangulations $T_{n_{j}}, j \geq 0$, of $T(\alpha)$ into $n_{j}$ triangles with

(a) $n_{0}<n_{1}<n_{2}<\ldots$;

(b) all triangulations $T_{n_{j}}$ are refinements of $T_{n_{0}}$; 


$$
\text { (c) } \operatorname{Max}\left(T_{n_{j}}\right)=O\left(\frac{1}{f\left(n_{j}\right)}\right) \text {. }
$$

We can perturb the vertices of the triangles in the triangulation $T_{n_{0}}^{\prime}$, so that we obtain a triangulation $T_{n_{0}}^{\prime \prime}$ of $T\left(\alpha^{\prime \prime}\right)$ which has the same combinatorial type as $T_{n_{0}}^{\prime}$ and whose vertices have all rational coordinates. We denote $p\left(\alpha^{\prime \prime}\right), p_{2}, \ldots, p_{m}$ to be the points in $\mathbb{R}^{2}$ that correspond to the vertices of the triangles in $T_{n_{0}}^{\prime \prime}$. In particular, $\alpha^{\prime \prime}$ is then rational, say $\alpha^{\prime \prime}=\frac{t_{n}}{u_{n}}$ with $\operatorname{gcd}\left(t_{n}, u_{n}\right)=1$. If $A_{i}^{\prime \prime}$ is the area of the triangle $\Delta_{i}^{\prime \prime}$ in the triangulation $T_{n_{0}}^{\prime \prime}$, then $\frac{A_{i}^{\prime \prime}}{A_{1}^{\prime \prime}}$ is also rational. Let

$$
\frac{A_{i}^{\prime \prime}}{A_{1}^{\prime \prime}}=\frac{A_{1 i}}{A_{11}}, \quad A_{1 i}, A_{11} \in \mathbb{N} .
$$

Since we do not require that $\operatorname{gcd}\left(A_{1 i}, A_{11}\right)=1$, we may assume wlog that the denominator $A_{11}$ is the same for all $i$.

The continued fraction representation of $\alpha^{\prime \prime} \in \mathbb{Q}$ is finite, say $\alpha^{\prime \prime}=\left[a_{1}, \ldots, a_{n}\right]$.

We now define $\alpha$ and the desired triangulation $T_{n_{0}}$ of $T(\alpha)$. To this end, we first recursively construct a suitable sequence of natural numbers $a_{n+1}, a_{n+2}, \ldots$, and define $\alpha$ as

$$
\alpha=\left[a_{1}, \ldots, a_{n}, a_{n+1}, a_{n+2}, \ldots\right] .
$$

We then define the desired triangulation $T_{n_{0}}$ of $T(\alpha)$ as the triangulation which is of the same combinatorial type as $T_{n_{0}}^{\prime \prime}$ and whose vertices have the positions $p(\alpha), p_{2}, \ldots, p_{m}$. This choice for the positions of the vertices will be possible, because we will construct $\alpha$ in such a way that it is 'close enough' to $\alpha^{\prime \prime}$, and since, by assumption, none of the points $p_{2}, \ldots, p_{m}$ lies on the edge between the points $p\left(\alpha^{\prime \prime}\right)$ and $(1,0)$. We denote the triangle in $T_{n_{0}}$ that corresponds to the triangle $\Delta_{i}^{\prime \prime}$ in $T_{n_{0}}^{\prime \prime}$ by $\Delta_{i}$. The area of the triangle $\Delta_{i}$ is denoted by $A_{i}$.

First, we define $a_{n+1}$ appropriately. By definition, $\frac{t_{n}}{u_{n}}$ is the $n$th convergent of $\alpha$. Let $\frac{t_{n-1}}{u_{n-1}}$ be the $(n-1)$ st convergent of $\alpha$. Both $\frac{t_{n}}{u_{n}}$ and $\frac{t_{n-1}}{u_{n-1}}$ are independent of the choice of $a_{n+1}, a_{n+2}, \ldots$. By Lemma $3.1(i)$, we have

$$
\left|\alpha-\frac{t_{n}}{u_{n}}\right| \leq \frac{1}{u_{n}\left(a_{n+1} u_{n}+u_{n-1}\right)} .
$$

Thus, if we choose $a_{n+1}$ sufficiently large, say

$$
a_{n+1}>N,
$$

then $T_{n_{0}}$ is indeed a triangulation of $T(\alpha)$ which has the same combinatorial type as $T_{n_{0}}^{\prime}$ (independent of the choice of the $a_{n+2}, a_{n+3}, \ldots$ ).

It follows from (12) that for $a_{n+1} \rightarrow \infty$, the number $\alpha$ converges to $\frac{t_{n}}{u_{n}}$, and hence $\frac{A_{i}}{A_{1}}$ converges to $\frac{A_{1 i}}{A_{11}}$ (of course, if neither $\Delta_{1}$ nor $\Delta_{i}$ has $p(\alpha)$ as a vertex, then we have $\frac{A_{i}}{A_{1}}=\frac{A_{1 i}}{A_{11}}$. Since $A_{1}$ is bounded from above and since $A_{1 i}$ is a constant, it follows that for a sufficiently large $a_{n+1}$, we have

$$
\left|\frac{A_{i}}{A_{1 i}}-\frac{A_{1}}{A_{11}}\right|<\frac{1}{f\left(B_{1}\left(A_{11}+\ldots+A_{1 n_{0}}\right)\right)},
$$


for all $i=1, \ldots, n_{0}$, where $B_{1}$ is chosen so that for all $i$, the triangle $\Delta_{i}$ can be triangulated into $B_{1} A_{1 i}$ triangles of equal areas. Now we choose $a_{n+1}$ so that it satisfies (13) and (14) and also

$$
a_{n+1}>u_{n}^{n-1} .
$$

Condition (15) will be used later to show that the number $\alpha$ is transcendental.

Suppose now that $a_{n+1}, \ldots, a_{n+j-1}$ have already been chosen. Then we define $a_{n+j}$ using the same basic idea that we have used to define $a_{n+1}$ - we merely replace the ap-

proximation $\frac{t_{n}}{u_{n}}$ of $\alpha$ by the $(n+j-1)$ st convergent $\left[a_{1}, \ldots, a_{n+j-1}\right]=\frac{t_{n+j-1}}{u_{n+j-1}}$, and we replace the triangulation $T_{n_{0}}^{\prime \prime}$ of $T\left(\alpha^{\prime \prime}\right)$ by the triangulation $T_{n_{0}}^{\prime \prime \prime}$ (of the same combinatorial type) of the trapezoid $T\left(\frac{t_{n+j-1}}{u_{n+j-1}}\right)$ whose triangles have their vertices at the points $p\left(\frac{t_{n+j-1}}{u_{n+j-1}}\right), p_{2}, \ldots, p_{m}$.

For sufficiently large $a_{n+j}, \frac{t_{n+j-1}}{u_{n+j-1}}$ approximates the number $\alpha$ again arbitrarily well, and if $\frac{A_{j i}}{A_{j 1}}$ are the quotients of the areas of the respective triangles in $T_{n_{0}}^{\prime \prime \prime}$, then, analogously to $(14)$, we have

$$
\left|\frac{A_{i}}{A_{j i}}-\frac{A_{1}}{A_{j 1}}\right|<\frac{1}{f\left(B_{j}\left(A_{j 1}+\ldots+A_{j n_{0}}\right)\right)},
$$

for all $i=1, \ldots, n_{0}$, where $B_{j}$ is chosen so that for all $i$, the triangle $\Delta_{i}$ can be triangulated into $B_{j} A_{j i}$ triangles of equal areas.

Further, analogously to (15), $a_{n+j}$ is chosen so that

$$
a_{n+j}>u_{n+j-1}^{n+j-2} .
$$

Since $\frac{A_{j i}}{A_{j 1}}$ does not have to be a reduced fraction, we may assume wlog that

$$
A_{j 1}+\ldots+A_{j n_{0}}>B_{j-1}\left(A_{j-1,1}+\ldots+A_{j-1, n_{0}}\right) .
$$

By Lemma 3.1 (ii), it follows from (15) and (17) that $\alpha$ is transcendental. The desired refinements $T_{n_{j}}$ of the triangulation $T_{n_{0}}$ of $T(\alpha)$ are now obtained by triangulating the triangle $\Delta_{i}$ into $B_{j} A_{j i}$ triangles of equal areas, for each $i=1, \ldots, n_{0}$. Condition (ii) (c) concerning the area discrepancy of $T_{n_{j}}$ is then satisfied because of (14) and (16). Moreover, (18) guarantees that the inequalities in (ii) (a) hold. This completes the proof.

\section{Acknowledgements}

We would like to thank Günter M. Ziegler for his encouragement to work on these types of problems and for stimulating discussions. 


\section{References}

[1] Aigner, M., Ziegler, G.M., Proofs from THE BOOK, Springer-Verlag, Heidelberg, 4th edition (2009).

[2] Du, Y., Ding, R., More on cutting a polygon into triangles of equal areas, J. Appl. Math. \& Computing, 17, No. 1-2, 259-267 (2005).

[3] Hardy, G.H., Wright, E.M., An introduction to the theory of numbers, Clarendon, Oxford (1968).

[4] Jepsen, C.H., Equidissections of Trapezoids, Amer. Math. Monthly 103, 498-500 (1996).

[5] Jepsen, C.H., Monsky, P., Constructing equidissections for certain classes of trapezoids, Discret. Math. 308, 5672-5681 (2008).

[6] Kasimatis, E.A., Dissections of regular polygons into triangles of equal areas, Discret. Comp. Geom. 4, No. 1, 375-381 (1989).

[7] Kasimatis, E.A., Stein, S.K., Equidissections of polygons, Discret. Math. 85, 281-294 (1990).

[8] Koch, H., Number Theory: Algebraic numbers and functions, Graduate Studies in Mathematics, AMS, Providence (1997).

[9] Koch, H., Pieper, H., Zahlentheorie, Deutscher Verlag der Wissenschaften (1976).

[10] Mansow, K., Ungerade Triangulierungen eines Quadrats von kleiner Diskrepanz, Diplomarbeit, TU Berlin, Germany (2003).

[11] Monsky, P., On dividing a square into triangles, Amer. Math. Monthly 77, 161-164 (1970).

[12] Monsky, P., Calculating a trapezoidal spectrum, Amer. Math. Monthly 103, 500-501 (1996).

[13] Roth, K.F., Corrigendum to 'Rational Approximations to Algebraic Numbers', Mathematika 2, 1-20 (1955).

[14] Stein, S.K., Cutting a polyomino into triangles of equal areas, Amer. Math. Monthly 106, 255-257 (1999).

[15] Stein, S.K., A generalized conjecture about cutting a polygon into triangles of equal areas, Discret. Comp. Geom. 24, 141-145 (2000).

[16] Stein, S.K., Szabó, S., Algebra and Tiling: Homomorphisms in the service of Geometry, MAA, Washington DC (1994).

[17] Su, Z., Ding, R., Dissections of polygons into triangles of equal areas, J. Appl. Math. \& Computing, 13, No. 1-2, 29-36 (2003).

[18] Ziegler, G.M., private communication (2010). 